\title{
Solution on the Identification of Computer Network Fault
}

\author{
Wei Ge, Lei Luo \\ Jiangxi Vocation College of Ahead Software, China
}

Keywords: Computer, Network fault, Type distinction, Identification method, Solving measures

\begin{abstract}
In recent years, computer technology and Internet technology has been obtained the rapid development, and has been widely used in all aspects of people's work, life and learning, however, in this process, the scope and depth of the impact for computer network risk is also increasing. This paper briefly introduces several common types of computer network fault at present, and discusses the specific identification method and solving measures in detail, hoping to help the students to better learn the course of computer network.
\end{abstract}

Even in the advanced science and technology, people still will inevitably encounter some faults in the actual process of using the computer, these problems can big and can small, if the problem is light, it will interfere with the normal conduct of work program, otherwise will affect the operation of the entire computer network, to bring different degree of loss for individuals or companies. Therefore, when we learn the computer network knowledge, in addition to master basic operation techniques, should also be able to accurately identify the reasons for all kinds of computer network faults, and take corresponding measures, to ensure that the computer network is in a steady state of operation at all times, to give full play to its due role.

\section{The main types of computer network faults}

\subsection{Physical fault}

Physical equipment fault is a common category in computer network faults, mainly includes the problems on the aspects of hub, network card, port equipment, circuit and so on.

\subsection{Hub fault}

Hub fault is a kind of fault category that has less probability in computer network, and its influence is mainly reflected in the weakening of the actual application effect for the network. In the actual use of the computer network, if there is a fault in the hub, the entire network will be disconnected and unable to function properly.

\subsection{Network card fault}

The fault of network card is mainly related to the usage of the host, if the using frequency of computer host is high and has been used for a long time, the network card is easily affected by external environment, such as card slot has dust, network card is loose and so on, all may lead to network card fault, affect the stable operation of the computer network.

\subsection{Port equipment fault}

In general, port equipment fault can be directly observed through the corresponding indicator light, if the light in a certain place is not bright, or the flashing is not stable, then can indicate that the corresponding port device has fault, we can quickly find this port, and to carry out the expansion and maintenance work.

\subsection{Circuit fault}

Circuit is an important part of computer network, is also one of the most easily malfunctioning equipment in the actual process of operation, such as electromagnetic interference and circuit damage all will affect the running efficiency and service quality of computer network. Once the 
circuit is damaged, the network cable of the computer will be disconnected, the information cannot be transmitted normally, and the user will have difficulty in obtaining the required information. When the circuit is disturbed by electromagnetic wave, the transmission network signal will be weakened, which leads to the loss of network communication quality. In general, the physical fault in the the computer network generally embodied in the above four categories, in the vast majority of cases, the identification and solving methods of the faults for these problems are simple, after systematic training, students can quickly master relevant knowledge point and operation skills.

\subsection{Logical fault}

A complete computer network system generally consists of hardware and software facilities, the so-called logical fault refers to the fault of computer hardware and software in the process of interconnecting and running-in. As far as it is concerned, the logic fault mainly occurs on the two main carriers, the host and the router, which account for more than $70 \%$ of the faults for the whole computer network.

\subsection{Router fault}

Router fault is usually caused by configuration problem, if the parameter setting is not appropriate, the IP address of the network will be repeated delivery, at the same time, the router port parameters of each node is abnormal, to lead to the occupy CPU process increased, to affect the operation quality of the entire computer network.

\subsection{Host fault}

Compared with the router fault, the fault problem of the host is much more complicated, no matter the fault point or the fault type all are far more than the logic fault of the router. At this stage, the host fault is mainly embodied in the network card driver, network protocol, network equipment, and other aspects, the fault forms are very various, need us to check the identification one by one in actual process, to find out the root cause of fault problem. In addition, if the computer network is attacked by the virus, it may cause the logic fault of the host.

\section{General identification and solution of computer network fault}

\subsection{The identification and treatment of network connection fault}

Computer network connection fault generally can be divided into two kinds, one kind is that twisted pair appears problem, another is that crystal head has a problem, when we recognize network connection fault, can be started from these two aspects. First, twisted pair is one of the most common medium in the current network transmission, in real life, cables are often exposed to the external environment, the possibility of being affected or damaged by a person as a factor is larger, such as wear, trample, liquid corrosion, etc. When the twisted pair is damaged, it will directly cause the running speed of computer network slow down and affect people's normal work and study. In the recognition of the twisted pair fault, the two ends of the twisted pair can be detected by means of wire measuring instrument and other tools, to judge whether the twisted pair is in normal working condition. If you find the phenomenon such as twisted pair appear rupture or breakage, need to change in time, and take the corresponding protective measures, to reasonably plan the network layout, to keep it away from the area of human activity, thus effectively reduce the effects of human factors to the twisted-pair, to reduce the happening of the network connection fault. Second, the crystal head is an important part to connect the Internet and computer, if the crystal head encountered the force in the connection of external is too large, it's easy to have a loose phenomenon, can also lead to network connection fault. Therefore, we are dealing with this kind of fault, can choose to use special tools such as line pressing pliers to press the cable crystal head tightly, to ensure there will be no loosening or falling off during the use. In addition, due to the special materials of crystal head, prone to oxidation phenomenon, can also affect the stability and speed of the computer network, so we should pay attention to regularly check the normal usage of crystal head, timely replace the crystal head which has oxidizing phenomenon, as much as possible 
to reduce the incidence rate of network connection fault.

\subsection{The identification and treatment of virus fault}

Virus is a great threat to computer network security, if the threat is light, it will tamper with the data, and interfere with the normal operation of the program, otherwise will leakage some important privacy and confidential information, to bring bigger loss for the enterprise and individual, even lead to total collapse for the entire computer network, can't put into normal use again. Therefore, we should pay more attention to the identification and processing measures of network viruses when we study network fault, to fully ensure that all computer software programs are in a safe network operation environment. In fact, even in the most secure computer network system, there are still some loopholes, this is also one of difficult problems in computer field so far, what we can do is to fill these loopholes as much as possible, to reduce the chances of virus invasion, or to take corresponding precautions ahead of time before the virus invades.

\subsection{The identification and treatment of resource sharing fault}

The main performance of network resource sharing fault is that the computer in the same LAN cannot access the shared resource, the computer host cannot access the native resources, etc. In the process of practical application, the probability of occurrence for the network resource sharing fault is low, when we meet this kind of problem, we can consider the setup of the computer first, and analyze whether the internal setup has a problem, in general, the identification and treatment methods of the network resource sharing problem are simple, students need to master the knowledge related to computer network attribute setting.

\subsection{The identification and treatment of router fault}

In the recognition of computer network fault, if there is a fault in the router, general is to replace a new router. If replaced a new router, the computer network will still be unable to function properly, indicating that the router itself is not a problem, and it may be that the router's port configuration is abnormal. In the case of troubleshooting, we can start by checking the configuration of static routing and dynamic routing, if find out the configuration errors, need to rethink its configuration, to ensure that the router is always in normal working condition. In case of network fault due to insufficient memory of the router, we need to expand the capacity of the router and upgrade its internal storage space to achieve the goal of improving the quality of network service. If the reality condition is limited, can't directly replace or upgrade existing router, need to rethink the planing of computer network system, change the original topology structure, to ensure the router's real memory can satisfy the demand of the network operation, thus effectively reducing the occurrence of network fault.

\subsection{The identification and treatment of electromagnetic interference fault}

Electromagnetic interference is one of the main reasons to cause the circuit fault and reduce the quality of service for computer network, most of the network signals are transmitted through the medium of the cable, and the anti-interference ability of the cable itself is not strong, is easy to be affected by external magnetic fields or electromagnetic waves, then to affect the quality of signal transmission, to cause the computer network fault. Therefore, to reduce the network fault of electromagnetic interference, we should select the cable material with strong anti-interference ability in the first line of equipment configuration, and carry out scientific and rational planning of the buried circuit, as far as possible to bypass or away from the magnetic field, reduce the interference by network signal transmission in the process, to ensure the quality of network communication services.

\section{Conclusion}

In summary, under the background of modern society, the application of computer network technology has brought great convenience to people's work and study, not only can significantly 
improve the efficiency of their work, also effectively promote the development of social economy. To give full play to the application advantages of computer network technology, first of all, we should fully understand the categories of common computer network faults, second, can be able to quickly determine the specific cause of the problems, and then take corresponding solutions to suit the remedy to the case, in order to guarantee the safe and stable operation of computer network, so as to effectively promote the normal conduct of the affairs, to promote the progress and development of the entire society.

\section{References}

[1] Jin Sun. Research on the treatment of computer network fault and network maintenance methods [J]. Electronic test, 2017(16).

[2] Weili Long. Application of network communication in solving network fault [J]. Light industry technology, 2017(09).

[3] Zhuo Chen. The security and its protective countermeasures of computer network information [J]. China health information management journal, 2011(6).

[4] Renjie Li. Analyze on the analysis and maintenance of computer network fault [J]. Information recording materials, 2017(11).

[5] Ping Wang. Discussion on computer network security technology and preventive measures [J]. Digital technology and application, 2014(4).

[6] Feng Jiang. The risk and control of computer network [J]. Electronic technology and software engineering, 2017(12). 